\title{
Acquisition and Analysis of Traffic Accident Scene Information Based on Laser Point Cloud
}

\author{
Zheng Yuanmao $^{1,2}$, He Yuanrong ${ }^{1, *}$, Wang Xiaorong ${ }^{1}$, Chen Qiujing ${ }^{1}$ \\ ${ }^{1}$ Big Data Institute of Digital Natural Disaster Monitoring in Fujian, Xiamen University of Technology, Xiamen, 361024, China- \\ yuanmaozheng@iue.ac.cn, 2012112001@xmut.edu.cn, xrwang24@163.com, cqj0818@outlook.com \\ ${ }^{2}$ Key Lab of Urban Environment and Health, Institute of Urban Environment, Chinese Academy of Sciences, Xiamen 361021, China
}

KEY WORDS: Laser point cloud, Traffic accident scene, DLG, Information acquisition, Analysis

\begin{abstract}
:
With the rapid development of the economy, the number of vehicles in China has increased rapidly, which has also brought about frequent ills in traffic accidents. How to improve the efficiency of on-site treatment of traffic accidents, and quickly and accurately conduct accident investigation and analysis is imminent. This paper was based on the point cloud to draw the accident scene DLG, and then used the local elevation difference method to automatically extract the point cloud data of the accident vehicle, and analyzes the vehicle speed calculation, the damage area measurement and the road surface flatness, as well as constructs the overall 3D scene of the accident scene. By analyzing the DLG of accident scene, the point cloud data and the constructed 3D model, which could quickly improve the efficiency of traffic accident investigation. The application results show that the method of information collection and rapid exploration of the accident site what based on the laser point cloud not only provides a basis for traffic accident treatment, but also effectively shortens the exploration time of accident site. At the same time, it cloud relieve the traffic congestion in a certain extent with the obvious results
\end{abstract}

\section{INTRODUCTION}

With the rapid development of the economy, the number of vehicles in China has increased rapidly. Cars not only have greatly brought the facility of people's travel, but also brought about the disadvantages such as traffic accidents. After the traffic accident, it not only causes the huge economic losses to both sides of the accident, but also causes the secondary accidents such as indirect loss of traffic congestion (Zhao et al, 2010). The research shows that the $80 \%$ reason of traffic congestion in China which was caused by the traffic accidents. How to quickly and accurately analyze and research the road traffic accidents is very imminent. Therefore, on-site information collection and exploration techniques for traffic accidents are very important. The traffic accidents generally occur in the harsh environments such as rainy, fog or in the night. The traditional manual measurement methods have many defects in accuracy, efficiency and personnel safety and so on, which were easily susceptible to rainy or nighttime lighting conditions such as the weather effects, the low measurement accuracy and the demand of a large number of manpower and material resources, as well as caused the traffic congestion (He et $\mathrm{al}, 2016)$. The ground 3D laser scanning technology is an advanced and automatic stereo scanning technology, which is also known as "Real Scene Copying Technology". It has noncontact, omnidirectional and high-precision to reconstruct the 3D point cloud model of target quickly, which could greatly save the manpower, the material resources and it could reduce the labor intensity (Zheng et al, 2003). This technology can also be applied to the environments where people cannot reach and may be more dangerous. It has broad application potential in the building monitoring, the traffic accident handing and the legal evidence collection.
13D laser scanning technology is a kind of rapid development of new technology for exploring the accident scenes since the middle and late 20th century. It could changes the traditional single-point acquisition into continuous automatic surface data Acquisition, which greatly improves the speed of survey. Since the beginning of the 21 st century, the foreign traffic accident scene information collection systems have begun to propose the on-site measurement methods from the perspective of positioning (Tang et al, 2013). Previous studies (Chen, 2006) proposed a systematic research for the accident site information which was based on the LeicaHDS2500 system in 2006; Chris used the Rigl LMS Z210 system to conduct a comparative research from the perspective of accident investigation (Chris, 2004); On April 12, 2017, the German Dortmund football team bus exploded, and the German police used the ground 3D laser scanner to quickly scan and acquire the information of the on-site accident (Tian, 2017). In China, Zhang et al used the characteristics of non-contact and high-precision of 3D laser scanner to quickly acquire the point cloud data of the accident site and accurately map the scene of the accident in 2007 (Zhang et al, 2007); In 2012, Ning et al constructed the 3D model and 3D real-life map of the accident vehicle which were based on the point cloud data, and they measured various distances and other information on the reallife map to restore the accident scene more realistically (Ning et al, 2012); Zhuang researched the splicing, denoising and reduction algorithms of point clouds, and he realistically constructed a 3D point cloud model of vehicles with curved features (Zhuang, 2013). These research works have achieved some good results, but so far, there is no statistical and analysis report on the vehicle speed estimation and the vehicle damage area which were based on point cloud.

\footnotetext{
* Corresponding author
} 
With the unremitting efforts of scholars at home and abroad, the application of this technology in the acquisition of accident scene information is more and more extensive $(\mathrm{Hu}$ 2003). This paper collects the information of traffic accident scene which was based on the laser point cloud, obtains the accident scene DLG, extracts the point cloud of the accident vehicle, and calculates the vehicle speed, statistics and analyzes the area of damage and the road surface flatness, as well as constructs the overall 3D scene of the accident scene.

\section{DATA ACQUISITION AND PRE-PROCESSING OF LASER POINT CLOUD}

\subsection{Data Acquisition of the Laser point cloud}

In this paper, the traffic accident scene information collection is simulating a traffic accident scene at the crossroads in the campus. There is no traffic lights, and the traffic flow is small, the roadside is green and dense. As well as there is visual line obstruction at the intersection where is belong to a typical scene that easy to occur the traffic accidents. After the traffic accident, there a lot of information were left in the scene generally, the road information such as the drive direction of the vehicle, and whether the road contains corners and intersections and so on; the accident vehicle information such as the vehicle types, the vehicle collision position and the vehicle damaged area and so on; the pedestrian or non-motor vehicle information such as the pedestrian or non-motorized vehicle information such as the pedestrian collision position, and the the remanding position of non-motorized vehicle etc. The traffic accident scene contains a wealth of various types of information. When using 3D laser scanning technology to survey the traffic accident scenes, it is necessary to formulate the corresponding on-site point cloud data collection scheme according to the actual situation of the site, and arranging the location of the scanner site reasonably and accurately to ensure the complete acquisition of the on-site data information, and it could not lead to the loss of part of the collected data. At the same time, due to the suddenness and the urgency of accident, it is necessary to quickly survey the complete surrounding environment and arrange the instrument scanning scheme to facilitate the scanning quickly and efficiently. After the on-site reconnaissance, the data collection of accident site was set up for a total of 3 stations, it used 15 minutes, and the amount of point cloud data is about $500 \mathrm{M}$. As show in figure 1, the FARO scanner is collecting scenes of a van and a motorcycle and the site of bicycle collision accident scene.

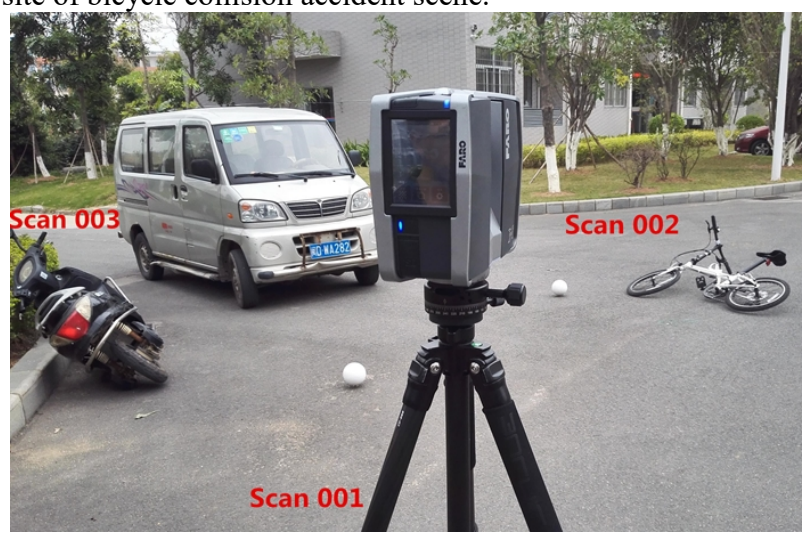

Figure 1. Scanner scans the scene of the accident

\subsection{Data Pre-processing of Point Cloud}

After the field data is collected, it is necessary to perform preprocessing such as the registration splicing and de-noising simplification of the point cloud data. Due to the scanning angle of view and the complexity of the target object, the scanning work of a single station cannot complete the complete data collection of the accident site. It is necessary to set up multiple sites to scan the target object at multiple angles. The point cloud data of different scanning sites are all based on the local coordinate system which were established by the respective scanners. The point cloud data needs to be registered and spliced into the same coordinate system, so that the point cloud data is spliced into a complete accident scene. The registration methods were based on target registration, based on manual extraction of feature point registration and the registration based on ICP algorithm. The main principle based on the ICP algorithm splicing is: for the point cloud data set to be spliced in the scanning site B and the point cloud of the reference site $A$, wherein the three-dimensional space represents the number of point cloud data included in the reference site A, indicating the number of point cloud data to be spliced in site B. In order to convert the coordinates of the point cloud data in the site $\mathrm{B}$ to be spliced into the reference site A, each point in the point set $\mathrm{B}$ needs to find the closest point in the site $A$ to form a point pair of the same name. In this way, the iterative calculation is continuously performed, and the error is converged to the threshold value for the iterative termination condition to perform iterative calculation, and finally the error function obtains the minimum rotation matrix $R$ and the translation matrix $T$ :

$$
f(R, T)=\frac{1}{N_{B}} \sum_{i=1}^{N_{B}}\left\|a_{i}-R b_{i}-T\right\|^{2}=\min
$$

Among them, $\left\|a_{i} R b_{i}-T\right\|$ it indicates the distance that between the $b_{i}$ converted to the coordinates in the base station A and its point of the same name. The target ball-based registration is based on the high reflectivity of a specific target, which could accurately extract the physical center of the reflected target ball as a registration point of the same name. The registration precision is high, the speed is fast, and the precision is up to millimeter; The extraction of feature points is mainly determined by the accuracy of feature points and the accuracy of extracting feature points. It is subject to subjective factors, the error is relatively large, the accuracy is low, and the accuracy is up to centimeter level. The registration speed based on the ICP algorithm registration is relatively slow, and the accuracy of the initial position of the point cloud and the conversion matrix are required to be high. If the initial parameter setting is unreasonable, the iterative calculation is a local optimal solution that is registered, rather than the overall registration accuracy. If the initial parameter setting is unreasonable, it is prone to iterative calculation as a local optimal solution of registration, rather than the overall registration accuracy.

Due to the fast splicing speed and high precision of the target ball-based registration, this paper adopts the registration and splicing method with target. In the registration and splicing process of point cloud data, the registration average error is less than $0.005 \mathrm{~m}$ as an exact match. After the traffic accident site point cloud data which was based on the target registration and splicing, the maximum registration deviation is $0.0034 \mathrm{~m}$, the minimum registration deviation is 0 , and the average registration error is $0.0028 \mathrm{~m}$, which satisfies the requirements 
that the complete registration error is less than $0.005 \mathrm{~m}$, the accuracy of the accident scene scanning and registration was relatively high. After the point cloud splicing is completed, the point cloud of the intersection, the road surface and the surrounding objects in the event of an accident needs to be extracted, and other point clouds are removed as noise point. When the noise point is removed, firstly dealing with the large areas of the surrounding area to reduce the amount of point cloud data; Secondly, the field-independent ground objects are removed, including the instrumental rotation jitter and the scattered scattered points generated by the moving objects in the three-dimensional view to accurately remove the data. That is the precise denoising. The site point cloud data after the preprocessing such as registration and denoising has threedimensional measurable coordinate information of spatial points, which can more accurately and intuitively acquire various spatial information such as on-site vehicles, roads and traffic signs for extracting and drawing. At the same time, the point cloud data can perform the three-dimensional scene rendering function, and the ground objects of different elevations are rendered and processed to facilitate the division of the ground objects. As shown in figure 2, the registered point cloud data is imported into HDmodeling for CAD2013 software for three-dimensional rendering, which can intuitively show that different elevations display different colors and improve the overall scene recognition.

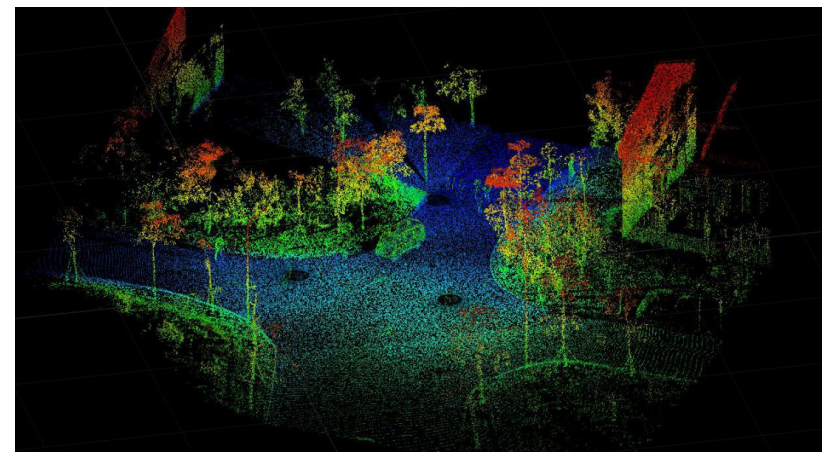

Figure 2. The rendering effect of point cloud data in the traffic accident scene

\section{DATA ANALYSIS}

3.1 DLG rendering based on point cloud data of accident scene

\subsubsection{Determining a reference coordinate system}

The plane rectangular coordinate system means an independent plane rectangular coordinate system if the coordinate origin is arbitrarily assumed. When plotting the accident scene digital line graph (DLG), the plane of the road can be used as a reference plane, and a plane rectangular coordinate system is established on this basis. According to the site situation, a fixed point with permanent marking is selected on the road and around the road as the reference point for the accident site orientation. The principle of selecting a traffic accident site reference point is: close to the traffic accident scene, easy to measure and draw, relatively fixed and hard to move. After the position of the reference point is determined, the $\mathrm{X}$ and $\mathrm{Y}$ axes are respectively drawn to establish a plane rectangular coordinate system. In this research, selecting the sewer cover above the intersection of the traffic accident site as a reference point, the following one side of the sewer cover takes the baseline as the origin of the coordinate system, the direction of the base line is X's direction, and perpendicular to the baseline direction is $\mathrm{Y}$ direction. The $\mathrm{Y}$ direction is just the north direction of the geographic coordinate system, which is easier to identify. The established reference coordinate system is shown in figure 3 . Selecting the sewer cover above the intersection of the traffic accident site as the reference point.

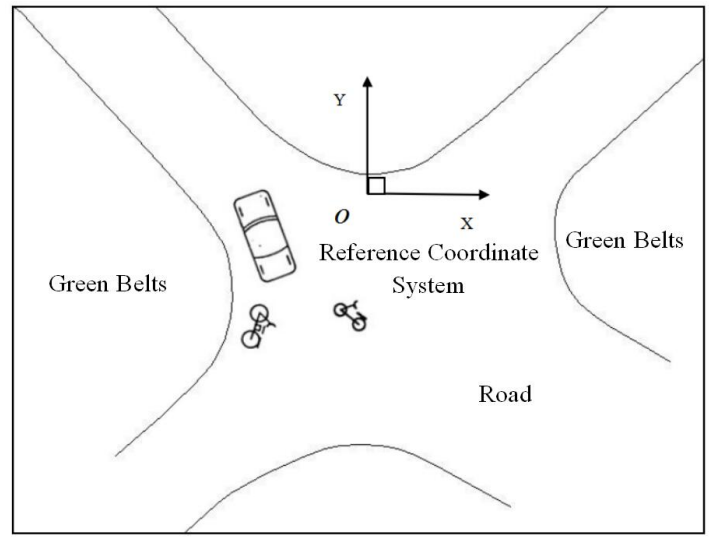

Figure 3. Establishing the coordinate system

\subsubsection{Map Production of the Road traffic accident scene}

Due to the road traffic accident scene map only needs to record the relative position, an independent coordinate system could be used when drawing the accident scene digital line drawing. According to the above-mentioned coordinate system, importing the point cloud data into the software, according to the difference of the point cloud data elevation and the degree of rendering, the HD modeling for CAD2013 software uses the points, lines, and surfaces of the DLG to draw the feature categories of different ground object attributes. In these point cloud data of the accident site, the main factors include the intersection of the accident, the position of the vehicle and the brake marks of the van, which was focus on the digital line drawing for further data measurement. Because the error of point cloud data is in the millimeter level, the precision is very high, and it meets the various data analysis requirements of the traffic accidents, and it would be the important basis for the information collection and surveying of traffic accident scenes. After the drawing is completed, the product is standardized and graphically described, and the DLG and data analysis of the final traffic accident scene are shown in figure 4. 


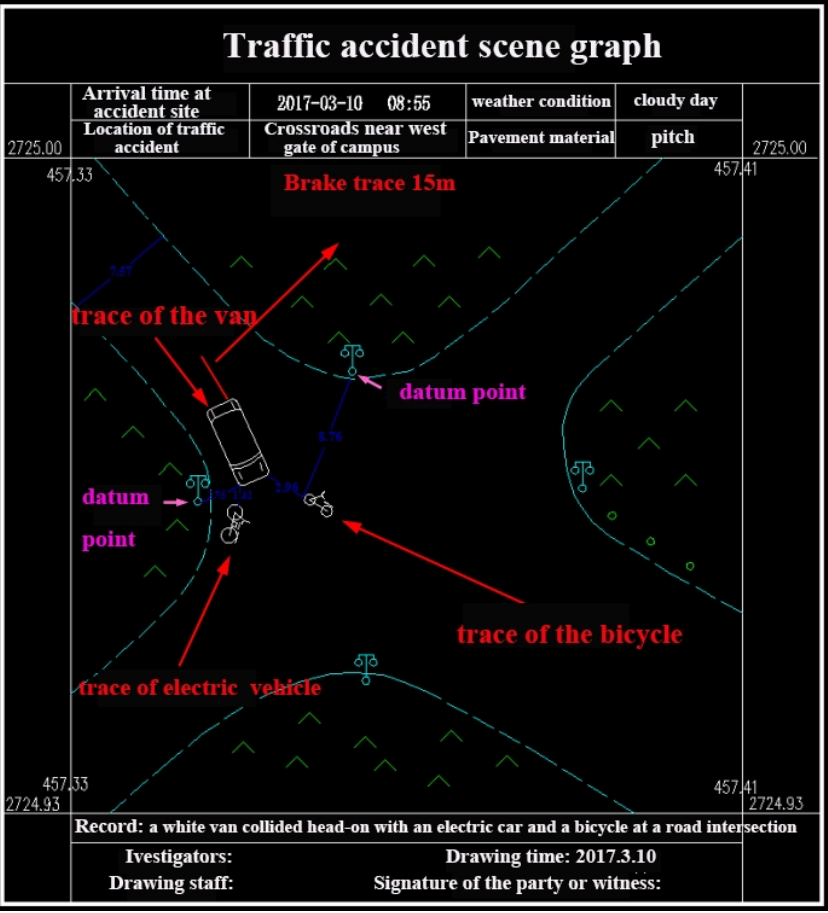

Figure 4 . The digital line drawing of traffic accident scene

From the DLG of this traffic accident scene, the following information could be obtained: (1) According to the brake marks, the wheel direction and other factors, the direction of the three vehicles can be judged; (2) Measuring the road driving of the van, and the road width is $7.57 \mathrm{~m}$, it indicates that when the road changed may be due to the situation that the car accident can not be avoided which was caused by the narrow road; (3) The length of the brake trace is drawn, the braking distance is $15 \mathrm{~m}$, which is convenient for providing the data support of the subsequent vehicle speed analysis; (4) After the accident, the distance between the electric vehicle and the van is $1.42 \mathrm{~m}$, and the distance between the bicycle and the van is $2.96 \mathrm{~m}$. It proved that the electric vehicle was a slight collision and the bicycle was seriously affected.

\subsection{Extraction and Analysis of the Accident Vehicle and the} Road Point Cloud

\subsubsection{Automatic Extraction of Vehicle Point Cloud}

After a traffic accident, it is urgent to analyze the accident vehicle, which is not only related to personal life safety, but also it cloud assess the severity of the accident and the cause of the accident. In order to analyze the damage situation of the vehicle more directly and objectively, as well as prevent other external factors from interfering with the extraction and analysis of the point cloud; In order to make the data more accurate and intuitive, it is necessary to extract the point cloud of the accident vehicle before analyzing the point cloud of the accident vehicle.

This paper adopts the partial elevation difference for the extraction of the traffic accident vehicle point cloud. Firstly all the point clouds are divided into grids(the laser point cloud data is about 28 meters long and 22meters wide, for which the paper is divided into 2822 grids); Then figure out the minimum point of each grid elevation; And the elevation difference is obtained for each point in the grid and the elevation minimum point; The threshold is set for the elevation difference, and first the threshold is set to $0.1 \mathrm{~m}$, as shown in figure 5 . The point cloud is divided into the accident vehicle point cloud and the ground point cloud, the accident vehicle point cloud is larger than the threshold value and the ground point cloud is smaller than the threshold value. The general elevation difference threshold is $0.1-0.2$ meters.

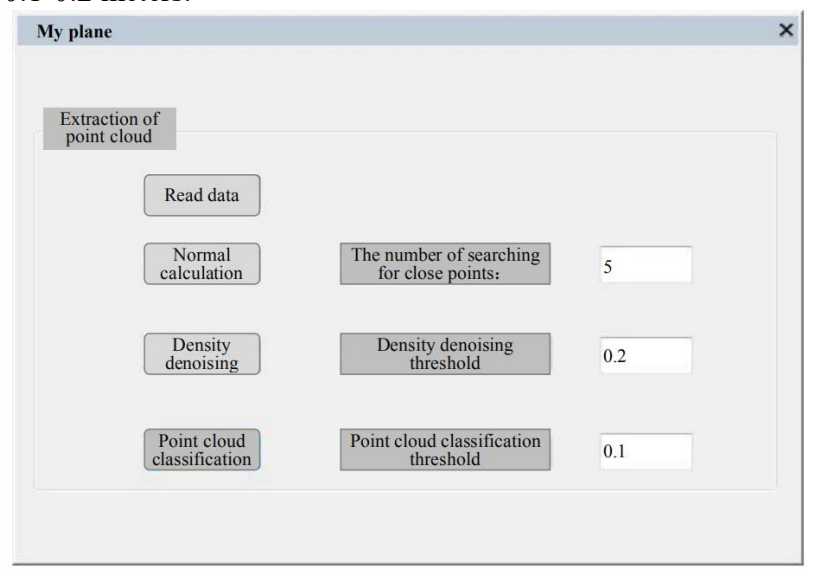

Figure 5. The program interface for automatic extraction of the accident vehicle point cloud

After a number of threshold comparisons, the extraction efficiency of the experimental data with the elevation difference threshold of $0.2 \mathrm{~m}$ is the best. The point cloud of the accident vehicle is shown in figure 6(a). From the classification effect map,due to the height of road ridge is generally not higher than $0.3 \mathrm{~m}$ (Nie, 2012), thus the road ridge is also divided into the accident vehicle points, and there are very few grounds points which are divided into the accident vehicle points. And so that the extracted accident vehicles are further extracted to remove the road ridge and the wrong point cloud. After many experiments, when the threshold value is 0.25 meters, the effect that obtained by this experiment data is the best. Finally, the point cloud of the accident vehicle is shown in figure 6(b), because the accident electric vehicle fell on the road, so that some of the roads adjacent to the electric vehicle were misclassified into the accident vehicles, and the extraction effect was good.

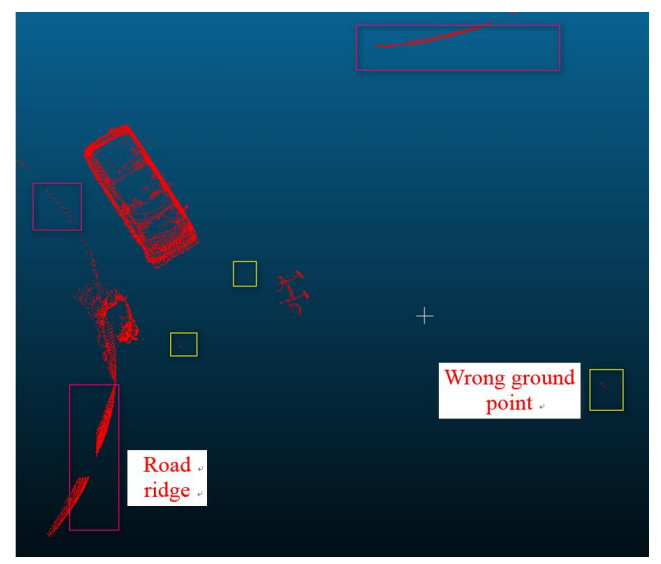

(a) Preliminary extraction 


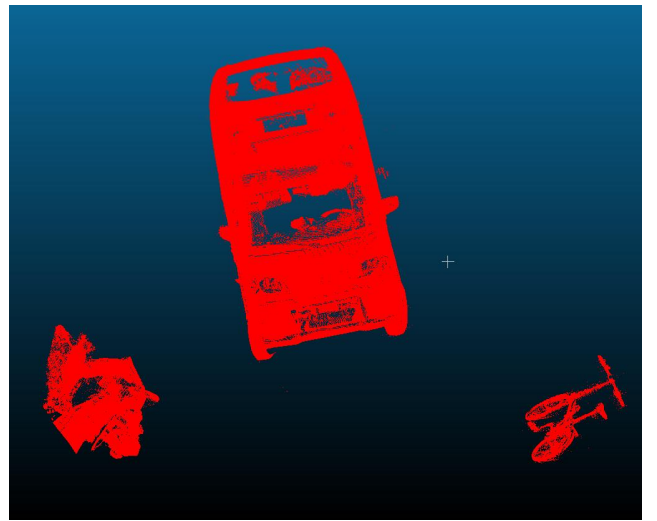

(b) Final extraction

Figure 6. Automatic extraction effect of accident vehicle point cloud

After extracting the specific point cloud information of the vehicle, the damage of the vehicle can be visually inspected, and the depression of the vehicle body can even get the steering of the tire, but the comprehensive traffic investigation and identification cannot be performed, and the following is a major analysis of the vehicle's braking traces and some damage situation of the vehicle itself and the road surface extraction.

\subsubsection{The Estimation of Vehicle Speed}

In the road traffic accidents, most of the vehicles in which the accident occurred during the movement, its motion belongs to uniform variable speed straight line motion. In this simulated traffic accident, when the driver responds to the emergency, the force during the emergency braking of the brake is always constant, so that the vehicle always performs the uniform linear motion during the accident. The uniform linear motion is divided into the the uniform acceleration linear motion and the uniform deceleration linear motion. The specific law follows the following formula (Dai, 2011):

The uniform acceleration linear motion:

$$
\left\{\begin{array}{c}
S_{t}=v_{0} t+1 / 2 * a t^{2} \\
v_{t}=v_{0}+a t \\
2 a x=v_{t}{ }^{2}-v_{0}{ }^{2}
\end{array}\right.
$$

The uniform deceleration linear motion:

$$
\left\{\begin{array}{c}
S_{t}=v_{0} t+1 / 2 * a t^{2} \\
v_{t}=v_{0}+a t \\
2 a x=v_{0}{ }^{2}-v_{t}{ }^{2}
\end{array}\right.
$$

Among them, $a$ is the acceleration, $v_{0}$ is the initial velocity, $v_{t}$ is the velocity at t seconds, $S_{t}$ is the displacement at $\mathrm{t}$ seconds. When the car adopts the braking process, it overcomes the maximum static friction force due to the problem of inertia, and starts to slide. The sliding distance is called the braking distance (Yang, 2006). During the braking process, the sliding friction formula it receives is:

$$
f=u N
$$

Where

$$
\begin{aligned}
& f=\text { friction } \\
& N=\text { the gravity of the car } \\
& u=\text { friction coefficient }
\end{aligned}
$$

According to the normative standard (Gong, 2006) in "GA/T643-2006 Typical Traffic Accident Form Vehicle Speed Technical Appraisal", the average deceleration during braking can be obtained by the following formula:

$$
a_{\max }=u g
$$

where $\quad g=$ gravity acceleration

$$
\mathrm{a}_{\max }=\text { average acceleration }
$$

Due to the vehicle is in a linear motion of uniform deceleration during braking, therefore, the driving speed can be derived based on the above formula:

$$
v_{0}=\sqrt{v_{t_{2}}+2 a s}
$$

In the formula, $s$ is the braking distance, which is the speed at stop, that is 0 . This formula is called "brake printing formula" , and is organized:

$$
v_{0}=\sqrt{2 u g s}
$$

According to the longitudinal friction coefficient table of the vehicle in "GA/T643-2006 Typical Traffic Accident Form Vehicle Speed Technical Appraisal", the value of $u$ can be known (Fang et al, 2013), as shown in Table 1:

Table 1. Vehicle tire longitudinal friction coefficient reference value table

\begin{tabular}{llcccc}
\hline & Material & \multicolumn{2}{c}{ Dry } & \multicolumn{2}{c}{ Damp } \\
\cline { 3 - 6 } & & $48 \mathrm{~km} / \mathrm{h}$ or less & $48 \mathrm{~km} / \mathrm{h}$ or less & $48 \mathrm{~km} / \mathrm{h}$ or less & $48 \mathrm{~km} / \mathrm{h}$ or more \\
\hline \multirow{2}{*}{$\begin{array}{l}\text { The concrete } \\
\text { table }\end{array}$} & New oavement & $0.80-1.00$ & $0.70-0.85$ & $0.50-0.80$ & $0.40-0.75$ \\
& Less wear on the road & $0.60-0.80$ & $0.60-0.75$ & $0.45-0.70$ & $0.45-0.65$ \\
& Large road wear & $0.55-0.75$ & $0.50-0.65$ & $0.45-0.65$ & $0.45-0.60$ \\
\multirow{2}{*}{ Asphalt } & New road & $0.80-1.00$ & $0.60-0.70$ & $0.50-0.80$ & $0.45-0.75$ \\
pavement & Less wear on the road & $0.60-0.80$ & $0.55-0.70$ & $0.45-0.70$ & $0.40-0.65$ \\
& Large road wear & $0.55-0.75$ & $0.45-0.65$ & $0.45-0.65$ & $0.40-0.60$ \\
\multirow{2}{*}{ Sandstone pavem } & Too much tar & $0.50-0.60$ & $0.35-0.60$ & $0.30-0.60$ & $0.25-0.55$ \\
Ash road & & $0.40-0.70$ & $0.40-0.70$ & $0.45-0.75$ & $0.45-0.75$ \\
Ice pavement & $0.50-0.70$ & $0.50-0.70$ & $0.65-0.75$ & $0.65-0.75$ \\
\multirow{2}{*}{ Snow pavement } & $0.10-0.25$ & $0.07-0.20$ & $0.05-0.10$ & $0.05-0.10$ \\
\hline
\end{tabular}


In the point cloud data, the brake marks of the vehicle are more obvious, and the point cloud of the brake trace is sparsely strip-shaped as a whole. As the brake trace point cloud data was shown in figure 5 . The point cloud data of the brake trace was extracted, and the braking distance was measured by AutoCAD 2013. In the simulated accident scene, the braking distance is 15 meters, as shown in figure 7.

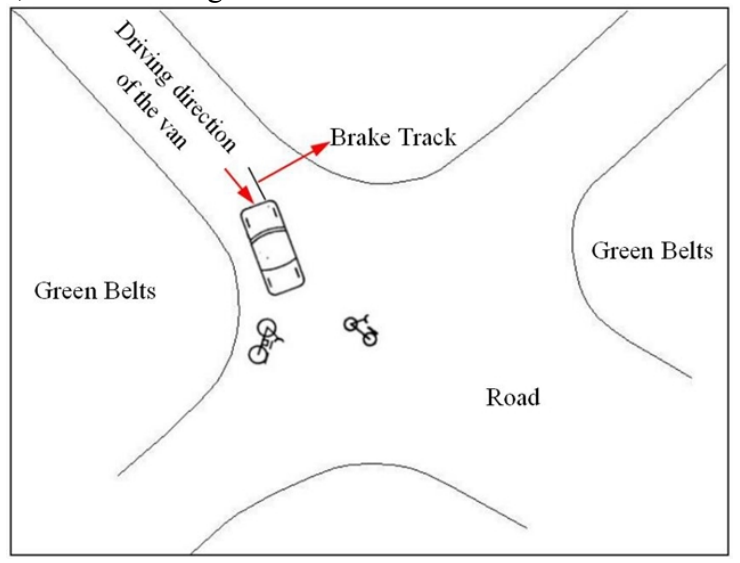

Figure 7. Acquisition of braking distance of vehicle

From the data in the table, the simulated accident site in this paper, the road is a dry asphalt pavement, it has little wear, the value of $\mu$ is about $0.55 \sim 0.7$, and the various values are substituted into the formula to roughly estimate the minimum driving speed of the vehicle is $13.845(\mathrm{~m} / \mathrm{s})=49.842(\mathrm{~km} / \mathrm{h})$, and the maximum is $14.345(\mathrm{~m} / \mathrm{s})=51.642(\mathrm{~km} / \mathrm{h})$.

\subsubsection{The measurement of vehicle damage area}

The outer surface of the vehicle is generally an irregular curved surface model. In the irregular damaged area of the accidental vehicle, the extracted accident vehicle point cloud data is segmented one by one and then imported into Geomagic Studio 2012 software to construct the vehicle model. After the vehicle point cloud data is imported into the software, the software performs the closed connection according to the distribution situation of point cloud, thereby constructing a three-dimensional model of the surface. After the threedimensional model of the accident vehicle is constructed, the damaged area of the accident vehicle can be drawn and selected in detail. According to the damaged areas drawn, the specific area size of the damaged area can be counted, which is close to the area calculated by the actual meter measurement, and the accuracy meets the actual requirements.

\subsubsection{The Statistical Analysis of Road Surface Point Cloud}

Not only the traffic accident site point cloud data is imported into FAROscene and Geomagic software for information extraction and analysis of accident vehicles. The MATLAB editing program can also be used to statistically analyze the smoothness of road pavement, and in order to further perform the complete and comprehensive extraction and analysis for the point cloud. When use a 3D laser scanner to scan the traffic accident scene, the point cloud data on the road surface is also be acquired. According to the coordinates of each point in the point cloud data set, a spatial plane is fitted, and the deviation of each point in the point set relative to the fitted spacial plane can be calculated, and the flatness of the road surface can be obtained (Guan et al, 2008; Cang et al, 2014).

Based on the MATLAB environment, the pavement point cloud flatness detection program mainly uses the function of computer to process data efficiently and quickly, and working over the point cloud data of the road surface. The program imports data into the program by loading point cloud data. The best spatial fitting plane is obtained according to the coordinates of each point in the point cloud data set. And calculating the distance from each point the the fit plane. Call the hist() function in MATLAB to color the distance and draw a histogram. The distance from each point to the fitting plane is visually analyzed for the smoothness of the inner surface of the road surface.

The plane fitting and statistical analysis program development of pavement flatness is carried out by MATLAB. The key codes are as follows:

$\mathrm{x}=\mathrm{x} 0+\mathrm{dx} 1 ; \%$ the coefficient of the fit plane $\mathrm{a}, \mathrm{b}, \mathrm{c}$

$\mathrm{d}=(1 / \operatorname{norm}(\mathrm{x})) *\left(\right.$ part_of_second ${ }^{*} \mathrm{x}+$ ones $\left.(139872,1)\right) ; \% 139872$ points to the fit plane

M0=sqrt(norm(d)^2/(139872-3));\% flatness, the value is positive for the bump, and the negative value for the pit

hist $(\mathrm{x}, \mathrm{n}) \%$ where $\mathrm{x}$ is a one-dimensional vector, the function of the function is to divide the interval between the minimum and maximum values in $\mathrm{x}$ by $\mathrm{n}$, the abscissa is the $\mathrm{x}$ value, and the ordinate is the number of the value

hist(d,50);\% distance distribution histogram

xlabel('distance d')

ylabel('number n')

\}

At the same time, in the statistical analysis of road point cloud using MATLAB, if the number of point clouds in the point cloud data is more than one million, the phenomenon of jamming will occur. When using the MATLAB program to measure the flatness of the road surface, the road point cloud data can be compressed and thinned, and the statistical rate can be greatly improved without affecting the point cloud distribution of the road condition. Therefore, in this experiment, the point cloud data of the middle area of the road is selected for statistical analysis. As shown in figure 8, the flatness statistics situation of the road point cloud are obtained. From the figure, the road surface flatness is good. Since the road point cloud data is thinned out, the amount of the statistical point cloud data is small.

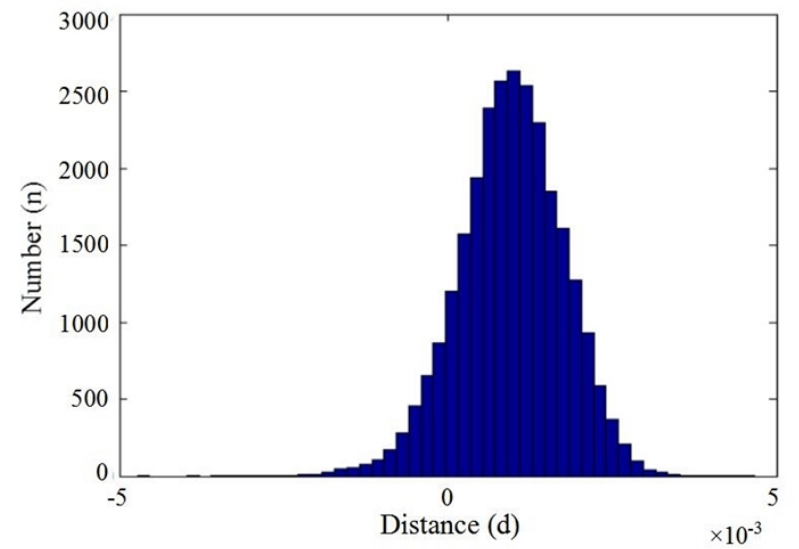

Figure 8 . The statistical histogram of point cloud distribution on road pavement 
3.3 Construction and Analysis of 3D Scenes of Accident Scenes

\subsubsection{Construction of the overall 3D scene}

Through the reconstruction of the $3 \mathrm{D}$ scene of the accident site as a whole, the accident scene can be reproduced in detail and completely, and the situation and severity of the accident can be quickly grasped. It is also an important basis for analyzing the scene of the accident. Based on the complete point cloud data of the accident scene, this paper can simulate and reconstruct the 3D scene of the accident scene. The traffic accident site point cloud data is imported into HD modeling for CAD2013 software. Firstly, the coordinate system is established, and the complete 2D line drawing and model construction of the surrounding scenes such as roads and buildings are extracted. Secondly, it is imported into the 3DMAX software and then carries out the fine construction of traffic facilities such as tree greening, street lamps and other traffic facilities and accident vehicle models at the scene of traffic accidents. It also carries out texture mapping and finally completes the reconstruction of the complete $3 \mathrm{D}$ scene of the accident scene. The accident scene can be analyzed intuitively and completely through the overall $3 \mathrm{D}$ scene of the accident scene. The overall three-dimensional scene map of the traffic accident scene is shown in Figure 9. From the picture, you can quickly and truly understand the roads, the buildings, the greening, the street lights and the specific location and the status of the accident vehicles after the collision, which could provide the important technical support for the accident analysis.

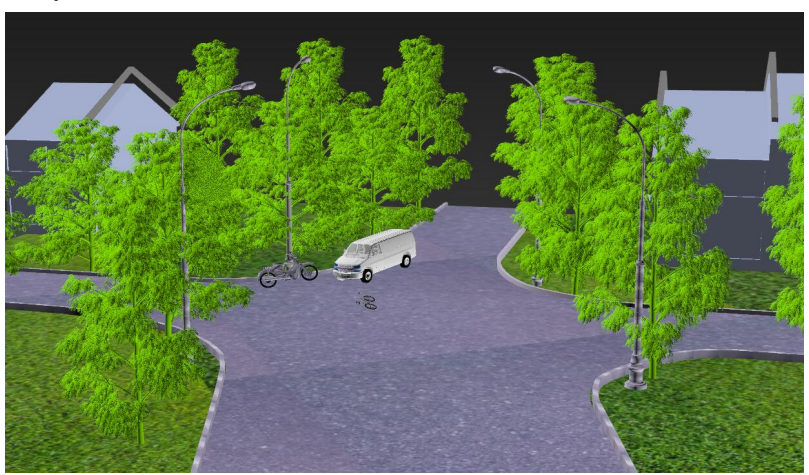

Figure 9. The construction of the overall 3D model of the traffic accident site

Through the three-dimensional scene of the accident scene mentioned above, it can be concluded that the accident vehicles are driven as follows: when the accident occurs, the van is in a high-speed driving state, and when the two target vehicles are seen, the emergency braking is performed, and the speed is too fast to stop on time. And the lower part of the car under the headlights hits the electric car, causing it to rush into the edge of the road; The bicycle under the left part of the car hits the bicycle. Due to the large impact force, the mass of bicycle is lower, so that it is hit a few meters away and falls to the center of the intersection, which belongs to a typical vehicle collision accident.

\subsubsection{Comprehensive Analysis of the Accident Site}

In summary, through the construction of the overall threedimensional model of the traffic accident site, the following conclusions can be concluded.

(1)In the aspect of road, the three-dimensional model analysis can obtain a wide view of the road surface, resulting in the slack of the driver of the van, no traffic lights, resulting in too fast speed to slow down the vehicle speed at the intersection and causing a car accident.

(2)In the aspect of vehicles, the electric cars and their bicycles are seriously damaged according to the survey. The electric car has already rushed into the grass, and the bicycle was directly hit by a few meters and fell to the center of the road, which actually restore the scene of the traffic accident.

(3)Through the overall three-dimensional scene of the accident, the survey personnel can quickly and intuitively understand and survey the accident scene, and can also be used to simulate the driving trajectory of the accident site to analyze the severity of the accident accurately and reasonably, and making the corresponding measures to reduce the accident. Finally, the overall 3D scene of the accident scene is constructed.

\section{CONCLUSION}

This paper mainly researches the collection and analysis of traffic accident scene information which was based on laser point cloud data. The DAG data of the accident scene is drawn by the point cloud data. Secondly, the point cloud data of the accident vehicle is automatically extracted by the local elevation difference method, and the vehicle speed calculation, the damage area measurement and the road surface flatness are statistically analyzed. Through the analysis of the 2D DLG data of the accident site and the $3 \mathrm{D}$ model data constructed, the cause of the traffic accident can be quickly and efficiently investigated. The application results show that the method of rapid exploration of the accident site based on laser point cloud not only provides the basis for traffic accident treatment and effectively shortens the time of accident site investigation, and relieves traffic congestion to a certain extent, with obvious results.

\section{ACKNOWLEDGEMENTS}

This research was supported by he National Natural Science Foundation of China (grant no. 41574011) and the Open Fund Programs of Big Data Institute of Digital Natural Disaster Monitoring in Fujian (grant no. NDMBD2018004)

\section{REFERENCES}

Cang Guihua, Yue Jianping, 2014. Plane fitting of point clouds based on weighted total least square. Laser Technology, 38(03):307-310.

Chris Hyslop, October, 2004. 3D Terrestrial Laser Scanners for Po-lice Mapping. A dissertation submitted for the degree of Bachelor of Surveying with Honours University of Otago, Dunedin New Zealand:65-78.

Chen Xian-dong, 2006. A 3D laser scanning system for traffic accident site based on VRS. Science of Surveying and Mapping, 31(6):153-155.

Dai Yaohui, 2011. The Analysis and application of vehicle speed identification method for road traffic accidents. Xi'an: Chang'an University.

Fang Lina, Yang Bisheng, 2013. Automated Extracting Structural Roads from Mobile Laser Scanning Point Clouds. Acta Geodaetica et Cartographica Sinica, 42(2):260-267. 
Gong Biao, 2006. Appraisal form of a typical traffic accident vehicle speed technical. China Standards Press.

Guan Yunlan, Cheng Xiaojun,Shi Guigang, 2008. A Robust Method for Fitting a Plane to Point Clouds. Journal of Tongji University (Natural Science), 36(07):981-984.

He Yuanrong, Zheng Yuanmao, Pan Huoping et al, 2016. Real Three-dimensional Modeling and Application of Complex Construction based on the Point Cloud Data. Remote Sensing Technology and Application, 31(6): 1091-1099.

$\mathrm{Hu}$ Zhigui, 2003. Key Techniques and Solutions to City Planning in True3D Environment. Journal of Geomatics, 28(4):36-38.

Yang Shengwen, 2006. Study on Speed Evaluation Methods for Vehicles Involved in Road Accidents. Xi'an: Chang'an University.

Zheng Dehua, Lei Weigang, 2003. The Technique of Land 3D Laser Scanning and Imaging Survey. Railway Air Survey, 29(2):26-28.

Zhao Hongrui, Wang Ganggang, Liu Xuekong, 2010. Compare on Traffic accident scene exploration technology. The second
Nie Lele, 2012. Information Processing Technology of Threedimensional Measurement System based on Phase Laser. Changchun: Changchun University of Science and technology.

NING Yipeng, LI Lei, PAN Weiguo, 2012. Discuss of traffic accident scene processing method based on 3D laser scanning technology. Technology Wind, (5):44.

Tian Yuan. Borussia Dortmund football team suffered bomb attacks in Germany. Guangming daily,2017-04-13(10).

Tang Kun, Hua Xianghong, Wei Cheng, et al, 2013. The Experimental Study of Buildings Deformation Monitoring Method Based on 3D Laser Scanning. Journal of Geomatics, 38(2):54-55.

"Surveying and mapping science frontier technology BBS" Papers selected:21-25.

Zhuang Shijun, 2013. Traffic Accident Reconstruction Based on 3D Laser Scanning Technology. Zhenjiang: Jiangsu University.

Zhang Xiaoyun, Jin Xianlong, Qi Wenguo, et al, 2007.Vehicle Crash Accident Reconstruction Based on Fem Neural Networks. Chinese Journal of Mechanical Engineering, 43(3):143-147. 